P210 (continued)

Outcome Measures and Analysis: The interviews were transcribed verbatim. Interviews were coded using descriptive and in-vivo strategies to develop a coding scheme. Constant comparison was used and inter-rater reliability was assessed. A matrix with diagnosis by child's eating issues and clinician's nutrition-perspective was created to analyze which strategies were used for which categories of issues.

Results: Strategies were categorized based on the five senses. Strategies related to touch were used more often with those were less independent while verbal strategies were used more often with children who were more independent. Clinicians' nutrition-perspectives were most indicative of which strategies were used. Verbal and physical food-appreciation strategies were used most often among those who focused on whole, unprocessed foods, while varying tastes and texture strategies were used more frequently among those who focused on an "all foods fit" approach.

Conclusions and Implications: These findings warrant further study to determine which feeding strategies are effective across the spectrum. This may assist clinicians and parents to improve mealtime behaviors and diet quality in this pediatric population.

Funding: None.

\section{P211 Food, Health \& Choices (FHC): How Perceptions of Maternal and Peer Energy Balance Behaviors Relate to Students' Own Behaviors}

Rachel Paul, MS, RD, rachel.paul@tc.columbia.edu, Teachers College, Columbia University, 525 West 120th Street, Russell Hall 414, Mailbox \#137, New York, NY 10027; H. Gray, PhD, RD; P. Koch, EdD, RD;

I. Contento, PhD; M. Burgermaster, MAEd, MS

Objective: Many health behavior theories implement social determinants, yet exactly how social factors influence energy balance-related behaviors (EBRBs) remains unclear. This study examines how children's perceptions of others' EBRBs compare to their own.

Design, Setting and Participants: Fifth grade students ( $\mathrm{n}=215 ; 10.8 \mathrm{y} ; 49.8 \%$ male) in four urban public schools participated in the delayed control FHC childhood obesity prevention curriculum and wellness policy intervention during the 2013-14 school year.

Outcome Measures and Analysis: Students completed an audience response system questionnaire pre- and postintervention which measured their EBRBs and those perceived of their peers and mothers. General linear models were constructed (SPSS v20) to examine how children's post-behaviors were explained by mothers' and peers' pre-behaviors, controlling for children's own pre-behaviors and gender.

Results: Students' higher perceptions of mothers' vegetable intake was related to increased vegetable frequency post intervention $(\mathrm{F}=4.586, \mathrm{P}=0.034)$. Also, perceptions of lower sweetened beverage intake by mothers resulted in lower post intervention intake of sweetened beverage $(\mathrm{F}=5.980, \mathrm{P}=0.016)$. Similarly, positive perceptions of mothers' behaviors led to longer bouts of moderate-vigorous activity $(\mathrm{F}=6.122$, $\mathrm{P}=0.014)$ and less recreational screen time $(\mathrm{F}=8.689$, $\mathrm{P}=0.004)$. Peers positively influenced fast food intake only $(\mathrm{F}=4.141, \mathrm{P}=0.044)$.

Conclusions and Implications: Children's perceptions of mothers' behaviors seemed to strongly influence both positive and negative EBRBs; those who were influenced by their mothers' behaviors experienced an amplified effect of the FHC intervention. Upper elementary school children, while likely experiencing increased effects of peer pressure, still seem to be heavily influenced by perceptions of mothers' EBRBs. Highlighting maternal influences is warranted in future obesity prevention studies.

Funding: USDA.

\section{P212 Convergent Validity of Resonance Raman Spectroscopy in Three to Five Year Old Children}

Taren Swindle,PhD, tswindle@uams.edu, University of Arkansas for Medical Sciences, 521 Jack Stephens Drive, Little Rock, AR 72205; L. Whiteside-Mansell, EdD

Objective: This poster presents data on the utility and validity of the Resonance Raman Spectroscopy (RSS) device for carotenoid assessment in children. This is the first study to explore the use of the device in children less than 5 years.

Design, Setting and Participants: RSS carotenoid values were obtained from 120 children in 12 Head Start classrooms. Parents completed a Food Frequency Questionnaire (FFQ) relative to 8 fruits and vegetables on a scale of 1 (Never) to $9(2+$ times/day) over the last month.

Outcome Measures and Analysis: The RSS carotenoid value was used to categorize children as above or below the national average $(23,000)$. ANCOVA analyses adjusting for child age and ethnicity were used to examine differences between RSS categories on each FFQ item. Covariate-adjusted bivariate correlations between groups were computed.

Results: For all FVs, children with above average RSS scores had higher FFQ scores. Differences between groups were significant or marginally significant for 5 of 7 FVs. Adjusted-bivariate correlations suggest a positive, linear relationship between RSS scores and FV consumption (range $r=0.06$ to $r=0.33$ ).

Conclusions and Implications: Previous studies have shown a convergence between dietary questionnaires and plasma carotenoid levels in adults (e.g., Lai et al., 2014). This study builds on emerging evidence that RSS may be a valid tool for dietary assessment in young children (Aguilar et al., 2014). Due to the difficulty in findings reliable sources to report on child diet and the need to limit intrusive measurements, this device could provide an objective measure to the study of early child nutrition.

Funding: USDA. 